\title{
A double-edged function of DDX3, as an oncogene or tumor suppressor, in cancer progression (Review)
}

\author{
YU HE $^{1}$, DAN ZHANG ${ }^{1}$, YANFANG YANG ${ }^{1}$, XIXI WANG $^{1}$, XINYU ZHAO $^{1}$, PENG ZHANG $^{2}$, \\ HONGXIA ZHU ${ }^{3}$, NINGZHI XU ${ }^{1,3}$ and SHUFANG LIANG ${ }^{1}$ \\ ${ }^{1}$ State Key Laboratory of Biotherapy and Cancer Center, West China Hospital, Sichuan University, \\ Collaborative Innovation Center for Biotherapy; ${ }^{2}$ Department of Urinary Surgery, West China Hospital, \\ West China Medical School, Sichuan University, Chengdu, Sichuan $610041{ }^{3}$ Laboratory of Cell and \\ Molecular Biology and State Key Laboratory of Molecular Oncology, Cancer Institute and \\ Cancer Hospital, Chinese Academy of Medical Sciences, Beijing 100021, P.R. China
}

Received July 8, 2017; Accepted December 29, 2017

DOI: 10.3892/or.2018.6203

\begin{abstract}
DEAD-box RNA helicase 3 (DDX3) is a highly conserved family member of DEAD-box proteins in all eukaryotes from yeasts to human beings. Accumulating studies have confirmed DDX3 has the ability to regulate different steps of RNA metabolism, including RNA splicing, RNA export, transcription and translation initiation. Moreover, DDX3 is involved in many biological processes, such as stress response, cell apoptosis, cell cycle regulation and virus infection. In recent years, DDX3 is getting increasing attention due to its essential roles in cancer progression. However, DDX3 role in cancer development is rather complicated. This review mainly focuses on the dual roles of DDX3 and DDX3mediated signaling pathways in multiple cancers. In addition, the interplaying causes for the controversial roles of DDX3 in cancer are discussed. So far several small molecular chemical compounds targeting DDX3 are also summarized from the anticancer activity to the clinical trials of DDX3 inhibitors.
\end{abstract}

Correspondence to: Dr Shufang Liang, State Key Laboratory of Biotherapy and Cancer Center, West China Hospital, Sichuan University, Collaborative Innovation Center for Biotherapy, 17 Section 3, People's South Road, Chengdu, Sichuan 610041, P.R. China

E-mail: zizi2006@scu.edu.cn

Abbreviations: HCC, hepatocellular carcinoma; HBV, hepatitis B virus; $\mathrm{HCV}$, hepatitis $\mathrm{C}$ virus; EMT, epithelial-mesenchymal-like transformation; HIF-1 $\alpha$, hypoxia inducible factor-1 $\alpha$; HRE, HIF responsive element; HPV, human papillomavirus; RFS, relapse-free survival; OS, overall survival; OSCC, oral squamous cell carcinoma; BRCA1, breast cancer 1; NHEJ, non-homologous end joining; PLGA, poly(lactic-co-glycolic acid)

Key words: DDX3, cancer, oncogene, tumor suppressor, signaling pathway, inhibitor

\section{Contents}

1. Introduction

2. DDX3 role in cancer: Tumor suppressor or oncogene

3. Multifunctions of DDX3-mediated signaling pathways

4. Factors contributing to functional divergence of DDX3

5. Potential anticancer drugs targeting DDX3

6. Future perspectives

\section{Introduction}

DEAD-box proteins, named by the amino acid motif D-E-A-D (Asp-Glu-Ala-Asp), are members of the largest family of RNA helicases (1). Moreover, the DEAD-box proteins are ATP-dependent RNA binding proteins and RNA-dependent ATPases, which play essential roles in rearranging RNA-RNA and RNA-protein interactions (2). The DEAD-box RNA helicase 3 (DDX3), is one highly conserved family member of DEAD-box proteins in all eukaryotes from yeasts to human beings (3).

The human genome code for two types of functional DDX3 genes, including DDX3X and its homolog DDX3Y (4). DDX3Y is located on the Y-chromosome, and plays particular roles of spermatogenesis and male fertility (5). While DDX3X is located on the X-chromosome and widely expresses in a broad range of organisms. In addition, DDX3 is ubiquitously reported to control pleiotropic physiological events in a variety of tissues (6). Thus, most research pays more attention to DDX3X (refer DDX3 to DDX3X in following text).

As an RNA helicase, DDX3 protein consists of 662 amino acids. Its crystal structure has been reported by protein crystallization and $\mathrm{X}$-ray diffraction analysis $(7,8)$. Accumulating studies have confirmed that DDX3 has the ability to regulate different steps of RNA metabolism $(9,10)$, including RNA splicing (11), mRNA export (12-14), transcription (15) and translation initiation $(16,17)$. DDX3 manipulates RNAs that range from rRNAs to mRNAs. However, DDX3 is considered to be a nuclear-cytoplasmic shuttling protein through CRM1-mediated export pathway or Tip-associated 
protein-dependent export pathway $(13,14)$. After transporting from the nucleus to the cytoplasm, DDX3 is prepared for translation, and eventually destroyed. The translation of DDX3 is controlled by activation of elF3 and cap-dependent translation or inhibition of elF4 $(16,17)$. At the same time, DDX3 is involved in many biological processes, such as stress response (18), cell apoptosis (19), cell cycle regulation (20) and embryogenesis (21). A loss of DDX3 induces an early embryonic lethality in mice (21).

In recent years, DDX3 is getting increasing attention due to its essential roles in cancer progression (22). Accordingly, DDX3 may be a new potential target for cancer biotherapy. However, the roles of DDX3 in cancer development are rather complicated. As a 'double-edged sword' gene, DDX3 either promotes cancer progression or acts as a tumor suppressor in some cancer types. In the present study, we summarize and discuss the dual roles of DDX3 and DDX3-mediated signaling pathways in multiple cancers. In addition, we also analyze the interplaying causes for the controversial roles of DDX3 in different types of cancer and sum up the potential anticancer drugs targeting DDX3.

\section{DDX3 role in cancer: Tumor suppressor or oncogene}

The biological roles of DDX3 in cancer development are conflicting. Its tumor promoting and suppressing effects have been broadly reported. Notably, the dual roles of DDX3 are reported not only in different types of cancer but also in the same type of cancer. The divergent roles of DDX3 in multiple cancer progression (Table I) are discussed in the specific cancer as follows.

Hepatocellular carcinoma. DDX3 has been shown to be essential for the replication of hepatitis B virus (HBV) and hepatitis $\mathrm{C}$ virus (HCV) which are two types of hepatitis virus usually linked to hepatocellular carcinoma (HCC) $(23,24)$. DDX3 acts as diverse roles in HCC development. DDX3 overexpression was observed in $\mathrm{HCC}$, and it was identified as a cellular transforming gene in hepatocarcinogenesis (25). In contrast, Chang et al found that a decreased expression level of DDX3 is present in HBV-positive HCC patients, but not in the HCV-positive ones (20). Based on the findings, they proposed a molecular mechanism of DDX3 where the inhibition of DDX3 upregulates cyclin D1 and downregulates p21 $1^{\text {waf1/cip1, }}$ and thereby promotes $\mathrm{S}$ phase entry to facilitate tumor cell growth. Other research demonstrated that DDX3 inhibits cell colony formation ability of $\mathrm{HCC} \mathrm{HuH-7} \mathrm{cells} \mathrm{by} \mathrm{upregulating}$ $\mathrm{p} 21^{\text {waf1/cip1 } 1}$ in a p53-independent manner (15). A recent study supports DDX3 as a tumor suppressor in HCC, since DDX3 downregulation promotes stem cell-like properties and tumorigenesis by silencing the tumor-suppressive miRNAs in HepG2 cells (26). In addition, the decrease of DDX3 correlates with poor prognosis of $\mathrm{HCC}$ patients.

Breast cancer. DDX3 exerts oncogenic roles in breast cancer. DDX3 upregulation can increase the ability of cell growth, proliferation and epithelial-mesenchymal-like transformation (EMT) in normal breast epithelial MCF10A cells (27). Similarly, HIF-1 $\alpha$ induces the transcriptional activation of DDX3 via binding to the HIF responsive element located in the
DDX3 promoter region in MCF10A cells (28). Moreover, DDX3 is overexpressed in a large series of breast cancer patients (29), and DDX3 upregulation is correlated with distant metastases of breast cancer (30). While DDX3 knockdown decreases tumor volume and metastasis in vivo (31). In addition, a combination treatment using DDX3 and PARP inhibitors induces cooperative therapeutic effects in BRCA1-proficient breast cancer (32). Thus, DDX3 drives breast cancer carcinogenesis and it can be a potential treatment target for breast cancer.

Lung cancer. DDX3 has dual roles, inhibiting or promoting carcinogenesis, in lung cancer. DDX3 seems to have antitumor activity in human papillomavirus (HPV)-positive lung cancer. HPV E6 decreases DDX3 transcription to synergistically suppress p21 expression, thereby low level of DDX3 leads to a poor relapse-free survival (RFS) in early-stage lung cancer and non-small cell lung cancer patients $(33,34)$. Furthermore, loss of DDX3 suppressed E-cadherin by MDM2/Slug axis to promote tumor progression and metastasis in HPV-infected lung cancer cells (34).

However, DDX3 is also reported to overexpress in lung cancer, and DDX3 upregulation is related with shorter survival for lung cancer patients (35). While DDX3 downregulation decreases colony formation in lung A549 cells. In addition, a small molecule inhibitor RK-33 by targeting DDX3 can efficiently inhibit lung cancer growth (35).

To date, DDX3 roles are variable in lung cancer. It is still likely that DDX3 should be considered as a drug target for lung cancer therapy.

Colorectal cancer. DDX3 functions in colorectal cancer are controversial. Several studies showed that DDX3 acts as a tumor suppressor in colorectal cancer. Knockdown of DDX3 increases cancer progression through Snail/E-cadherin pathway in colon cancer cells (36). In addition, colorectal cancer patients with low DDX3 expression have poor prognosis and more frequent distant metastasis (36).

In contrast, several groups obtain different conclusions of the role of DDX3 in colorectal cancer (37-40). In KRAS-mutated colon cancer cells, DDX3 promotes cell invasion and the nodule formation of xenograft lung tumor due to $\beta$-catenin/TCF activation through the CK1ع/Dvl2 axis (37). In addition, DDX3 enhances KRAS transcription and activates the following $\beta$-catenin/ZEB1 signaling to promote KRAS-mediated tumor invasion (38). Accordingly, the colorectal tumor growth and metastasis mediated by KRAS gene mutations seem to require $\beta$-catenin/TCF activation by DDX3. While in KRAS-wild-type colorectal cancer, DDX3 promotes cell aggressiveness through the KRAS/HIF-1 $\alpha /$ YAP1/SIX2 cascade (39). Inhibition of DDX3 by a small inhibitor RK-33 reduces cell proliferation and causes a G1 arrest in colorectal cancer cells (40). In addition, a high DDX3 expression leads to poor clinical overall survival (OS) for colorectal cancer patients (37-40).

The conflicting role of DDX3 in colorectal cancer needs to be further explored. So far, it is inaccurate to predict survival outcomes of colorectal cancer patients only using the protein DDX3 due to its multiple functions of DDX3 in cancer development. It seems more feasible to jointly analyze several proteins, including DDX3 and its associated molecules, with colorectal cancer progression and prognosis. 


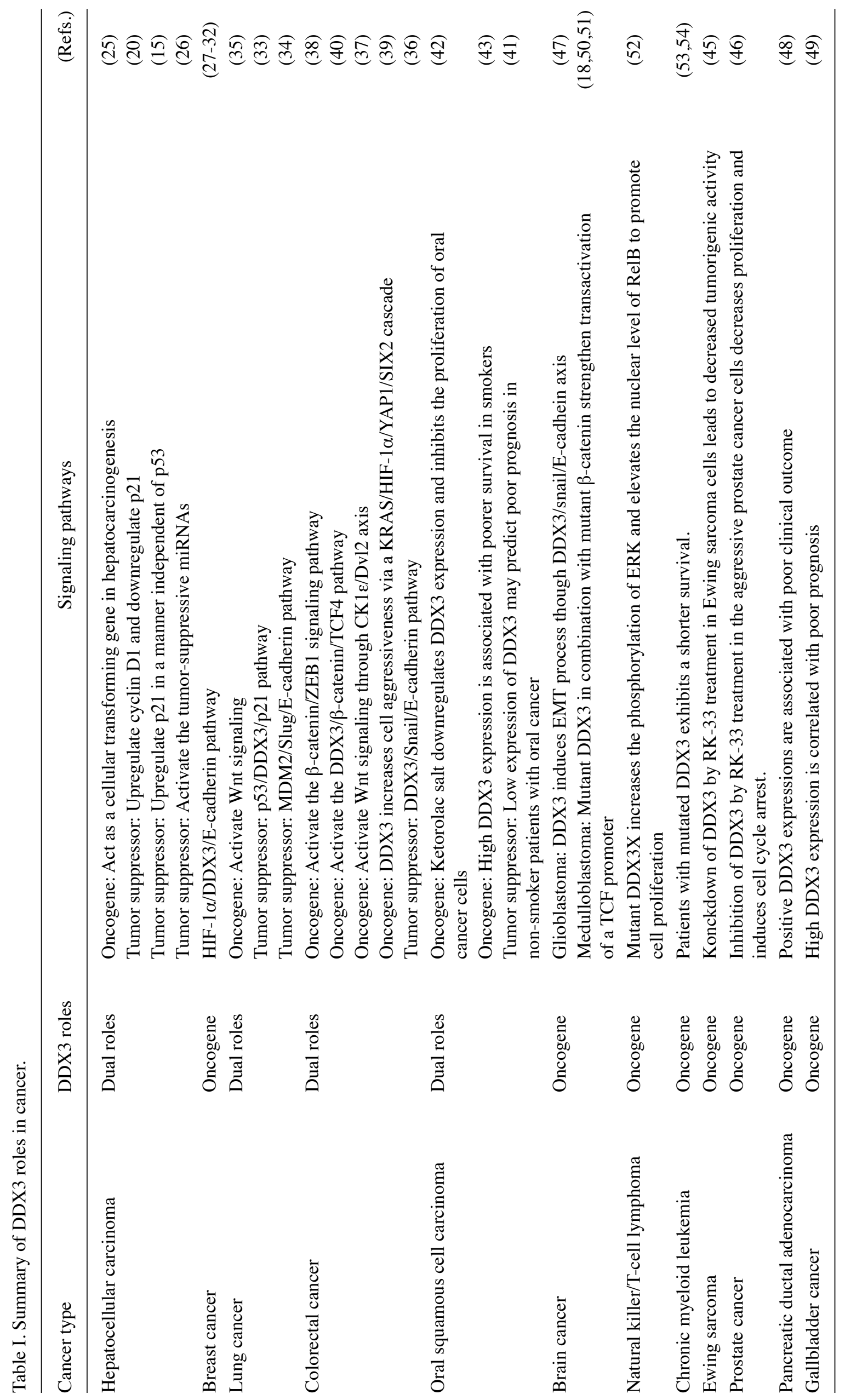


Oral squamous cell carcinoma. Notably, DDX3 has also been found to play divergent roles in oral squamous cell carcinoma (OSCC). A low/negative DDX3 expression is correlated with a poorer OS, notably in non-smoker patients with OSCC (41). This review demonstrates that DDX3 acts as a tumor suppressor, and it is probable to be an independent survival predictor in non-smoker patients with oral cancer.

Conversely, different conclusions claim that DDX3 is an oncogene to promote the progression of OSCC $(42,43)$. DDX3 expression is not correlative to survival in head and neck squamous cell carcinomas on the whole (43). However, a high DDX3 expression is associated with poorer survival in smokers. There are multiple reasons for explaining the different effects of DDX3 in oral cancer. One possible key factor is that smoker patients more often have HPV-negative tumors and non-smokers more frequently have HPV-positive tumors. DDX3 is increased in response to cigarette smoke exposure, whereas it has different roles in virus infection. Furthermore, DDX3 mutations have been found in head and neck cancers (44), which is partially responsible for the complexity of DDX3 roles.

Other cancers. DDX3 is also usually increased in the Ewing sarcoma (45), prostate cancer (46), glioblastoma (47), pancreatic ductal adenocarcinoma (48) and gallbladder cancer (49), in which a positive DDX3 expression is linked to a poor clinical outcome. However, the molecular mechanism of DDX3 exerting oncogenic functions in these cancers requires further study and discussion. Inhibition of DDX3 expression by inhibitor RK-33 leads to decreased tumorigenic activity of Ewing sarcoma cells and xenografts (45). Importantly, an in vivo combination treatment of RK-33 and radiation obtain synergistic inhibiting effects on prostate tumor growth (46). RK-33 alters cellular proteome, particularly proteins related to DNA damage repair, protein translation and proteasome function.

Exceptionally, the mutant DDX3 has been confirmed to have oncogenic functions in medulloblastoma $(18,50,51)$, natural killer/T-cell lymphoma (52) and chronic lymphocytic leukemia $(53,54)$. Mutations of DDX3 are identified in majority of medulloblastoma through whole-exome hybrid capture and deep sequencing. The mutant helicase domains of DDX3, in combination with a mutant $\beta$-catenin, strengthen transactivation of a TCF promoter and enhance cell viability in medulloblastoma (50). DDX3 mutants exhibit less RNA-unwinding activity and loss of depressing effects on cell cycle progression in NK cells, while the wild-type DDX3 inhibited the phosphorylation of ERK and reduced the nuclear level of RelB to inhibit cell proliferation (52). However, lymphocytic leukemia patients with DDX3 mutations have a poor prognosis and a shorter survival (53). Notably, DDX3 mutants are more frequently detected in cases relapsed after therapy (54). Therefore, the mutation of DDX3 is an essential factor leading to tumorigenesis. DDX3 has potential to be a new therapeutic target for natural killer/T-cell lymphoma.

\section{Multifunctions of DDX3-mediated signaling pathways}

The double-edged functions of DDX3 are partially due to multiple DDX3-mediated cell signaling pathways in different cancers. We summarize several DDX3-involved signaling pathways to promote or inhibit carcinogenesis and tumor development (Figs. 1-4). Cross-talk between different signaling pathways also contributes to the complexity of DDX3-modulated cellular activities.

DDX3-mediated signaling pathways to promote cancer progression

Wnt/ $\beta$-catenin pathway. The DDX3/Wnt/ $\beta$-catenin signaling pathway plays a pivotal role in tumor invasion. DDX3 directly binds to CK1ع to stimulate its kinase activity, and further promotes phosphorylation of the scaffold protein dishevelled (Dvl), thus facilitating $\beta$-catenin translocation into nucleus in a Wnt-dependent manner during the development of mammalian cells (55). Similarly, DDX3 acts as a subunit of CK1ع and phosphorylates Dvl2 to activate $\beta$-catenin/TCF signaling, and finally enhances tumor invasion in colorectal cancer (37). In addition, DDX3 increases protein levels of both Rac1 and $\beta$-catenin, and thereby promotes Wnt signaling to modulate cell adhesion, migration and metastasis (56). DDX3 mutations accompanied with mutant $\beta$-catenin increase transactivation of a TCF promoter and augment cell viability in medulloblastoma (50). Overall, these findings provide new insight into inhibiting cancer cell migration by targeting DDX3/Wnt/ $\beta$-catenin pathway.

DDX3/Snail/E-cadherin pathway. The EMT process, along with E-cadherin downregulation and Snail upregulation, accelerates the invasion and metastasis of cancer cells $(57,58)$. Although DDX3 is related to the Snail regulator GSK3 $\beta(59,60)$, DDX3 has the opposite effect on EMT in breast cancer and colorectal cancer cells. DDX3 knockdown downregulates Snail levels and decreases cell proliferation and migration in breast cancer MCF-7 cells (47). However, the inhibition of DDX3 induces the EMT process, with upregulation of Snail and decrease of E-cadherin in colorectal cancer DLD-1 and HCT116 cells (36).

HIF-1 $\alpha / D D X 3 / E$-cadherin pathway. DDX3 promotes oncogenesis in breast cancers through HIF-1 $\alpha / \mathrm{DDX} 3 / \mathrm{E}$-cadherin pathway $(28,29)$. Under hypoxic conditions, HIF-1 $\alpha$ binds to the HRE located in the DDX3 promoter region to induce the transcriptional activation of DDX3. Subsequently, upregulation of DDX3 represses E-cadherin expression, thus inducing the EMT process to facilitate breast cancer invasion.

KRAS/HIF-1 $\alpha / Y A P 1 / S I X 2$ cascade. In KRAS wild-type colorectal cancer, DDX3 increases cell aggressiveness via a DDX3/KRAS/ROS/HIF-1 $\alpha /$ DDX3 cascade feedback loop. ROS generation by DDX3-mediated KRAS expression promotes YAP1 transcription through increasing HIF-1 $\alpha$ bound to YAP1 promoter. Finally the YAP1/SIX2 axis results in the DDX3-induced cell invasiveness (39).

DDX3-modulates signaling pathway to inhibit tumorigenesis MDM2/Slug/E-cadherin pathway. DDX3 plays a tumor suppressor role in non-small cell lung cancer cells through the MDM2/Slug/E-cadherin pathway (34). DDX3 loss is induced by 553 knockdown and HPV E6 overexpression in lung cancer A549 cells. DDX3 loss inhibits MDM2 transcription via 


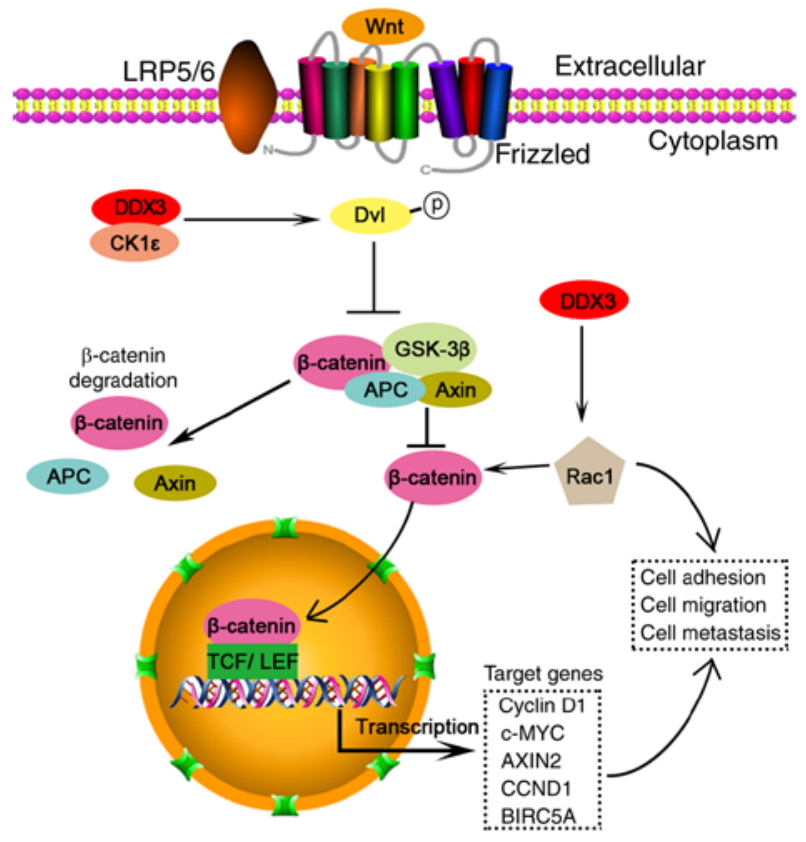

Figure 1. DDX3 acts as an oncogene through Wnt/ $\beta$-catenin pathway in colorectal cancer cells. DDX3 interacts with CK1 1 to form a complex, which phosphorylates dishevelled (Dvl). Dvl phosphorylation inhibits the generation of a complex of axin, GSK3 $\beta$, APC and $\beta$-catenin. The complex induces $\beta$-catenin degradation. In contrast, Dvl phosphorylation increases free $\beta$-catenin, which translocates from cytoplasm to nucleus. The nucleus $\beta$-catenin could interact with two major transcription factors, the T-cell factor (TCF) and lymphocyte enhancer factor (LEF), and regulate multiple gene transcription, such as cyclin D1, c-MYC, AXIN2, CCND1 and BIRC5A In contrast, DDX 3 elevates Rac1 expression and thereby increase $\beta$-catenin stability and its signaling. Racl and/or $\beta$-catenin can regulate cell adhesion, migration and metastasis.

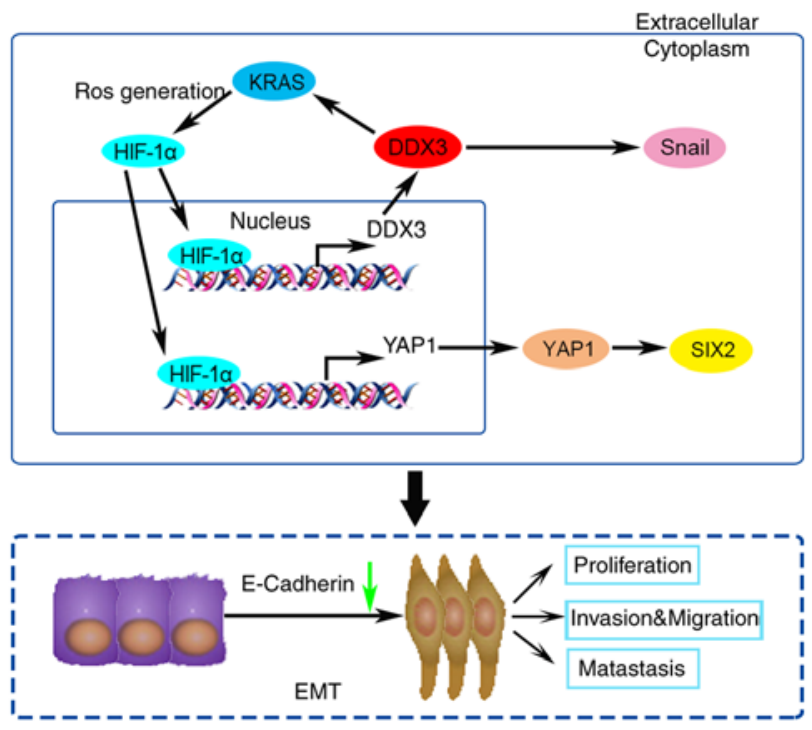

Figure 2. DDX3 plays oncogenic roles to improve the EMT process in colorectal and breast cancer cells. In breast cancer cells, DDX3 increases Snail expression, which decreases E-cadherin levels, and thereby promotes the EMT process. In contrast, in breast cancer cells, HIF-1 $\alpha$ binds to the HIF responsive element located in the DDX3 promoter region to induce the transcriptional activation of DDX3. Subsequently, upregulation of DDX3 represses E-cadherin expression, thus inducing the EMT process to facilitate cell invasion. In addition, in KRAS wild-type colorectal cancer, DDX3 increases cell aggressiveness via a DDX3/KRAS/ROS/HIF-1 $\alpha /$ DDX3 cascade feedback loop. ROS generation by DDX3-mediated KRAS expression promotes YAP1 transcription through increasing HIF-1 $\alpha$ bound to YAP1 promoter. Finally the YAP1/SIX2 axis induces DDX3-induced the EMT process.

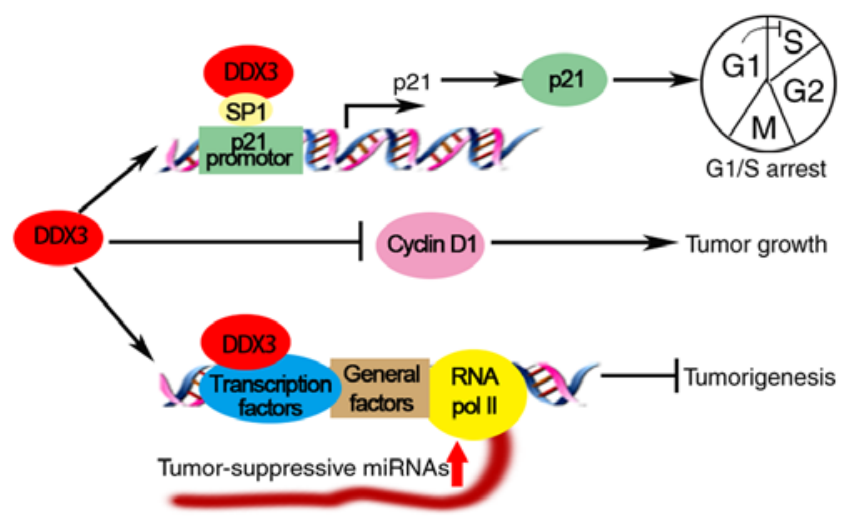

Figure 3. DDX3-mediates signaling pathways to suppress hepatocellular cancer cell growth. DDX3 interacts with Sp1 to increase the promoter activity of p21 regardless of p53 status, thus leading to S phase arrest. DDX3 also downregulated cyclin D1 to inhibit tumor growth. In addition, DDX3 could interact with transcription factors on promoter regions of tumor-suppressive miRNAs, such as miR-200b, miR-200c, miR-122 and miR-145, and activate the expression of miRNAs, thus suppressing tumorigenesis in liver cells.

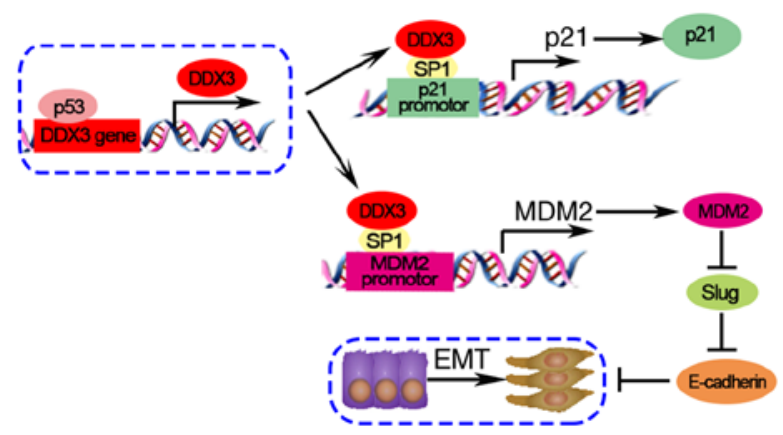

Figure 4. DDX3-mediated signaling pathways to suppress lung cancer cell growth. DDX3 increases MDM2 transcription by inducing SP1 binding to the MDM2 promoter, and MDM2 activation inhibits Slug expression. Then, MDM2 improves E-cadherin expression to inhibit tumor malignancy via mediating degradation of Slug in lung cancer cells. DDX3 could interact with Sp1 to enhance the promoter activity of p21 to function as a tumor suppressor.

reducing SP1 binding to MDM2 promoter. A previous study has demonstrated MDM2 improves E-cadherin expression via mediating degradation of Slug in lung cancer cells. While knockdown of MDM2 upregulates Slug and downregulates E-cadherin to promote tumor malignancy (61). In conclusion, in low DDX3-expressing cancer cells, DDX3 loss may enhance the tumor progression and metastasis through the MDM2/Slug/E-cadherin signaling axis.

p53/DDX3/p21 pathway. DDX3 interacts with Sp1 to upregulate the promoter activity of p21 regardless of p53 status in Huh-7 human hepatocellular cell line (15). DDX3 overexpression elevates p21 and decreases cyclin D1, leading to S phase arrest (20). In addition, DDX3 is regulated by p53, which enhances p21 transcription (33). Overall, DDX3 has functions as a tumor suppressor through the p53/DDX3/p21 signaling pathway.

DDX3/tumor-suppressive miRNA pathway. Accumulating research has demonstrated that microRNAs (miRNAs) 
exhibited tumor suppressive activity in cancer progression $(62,63)$. DEAD-box RNA helicase can involve in miRNA biogenesis (64). DDX3 is hypothesized to interact with transcription factors on promoter regions of tumor-suppressive miRNAs, such as miR-200b, miR-200c, miR-122 and miR-145, which possibly activates the expression of these miRNAs, thus, suppressing cancer stem cell properties and tumorigenesis in liver cells (26). Moreover, DDX3 interacts with Drosha/DGCR8 complex (miRNA microprocessor) as a novel RNA binding protein and affects pri-miRNA processing activity, and eventually promotes mature miRNA expression level to control cancer development (65). The DDX3-mediated miRNA regulation is promising to pave new strategy to explore its multiple functions.

\section{Factors contributing to functional divergence of DDX3}

Briefly, as an oncogene or tumor suppressor, DDX3 has a double-edged function in cancer occurrence and progression. Notably, even though in the same type of cancer, DDX3 roles are controversial. We discuss below the possible factors to confer many-sided abilities for DDX3.

Viral infection. DDX3 has been demonstrated to be an important component of the innate immune response against viral infections (66). DDX3 has a central place in virus-associated cancers for its proverbial function in viral replication. For instance, DDX3 expression level is decreased in HBV-positive, but not in the HCV-positive HCC patients (20). More importantly, DDX3 has been shown to promote $\mathrm{HCV}$ virus replication through its interaction with $\mathrm{HCV}$ core protein, but it interferes with $\mathrm{HBV}$ replication by binding to the HBV polymerase and inducing IFN- $\beta$ production (66). Therefore, DDX3 exerts specific functions on different types of virus infection in HCC development.

Although DDX3 has some relation with HPV infection, the molecular mechanism of HPV interaction with DDX3 is little known in HPV-infected cervical cancer, lung cancer $(33,34)$ and OSCC $(41,43)$. However, the interaction of DDX3 with virus protein was discovered to involve innate immune response against virus infection by targeting with TBK1 to induce IFN- $\beta$ production $(67,68)$. Moreover, DDX3 has been demonstrated to regulate $\mathrm{NF}-\kappa \mathrm{B}$ signal pathway $(69,70)$. DDX3-modulated immune response signaling still needs to be clarified in the virus infection-associated cancers.

Gender differences. DDX3 is located on chromosome X, and it is preferentially mutated in males. DDX3 can escape $\mathrm{X}$-inactivation, which may protect females from complete functional loss by a single gene mutation $(20,71)$. Moreover, DDX3 expression is closely associated with living habits (41), including smoking, alcohol consumption and other habits, which are more frequent in males than in females. For instance, a lower DDX3 level is more frequently present in male patients with HCC or OSCC than that in females $(20,41)$.

Other protein interactions. DDX3 interacts with variable proteins to involve in different signaling pathways, which also determines its function as tumor suppression or oncogenic role in cancer progression. For example, DDX3 exerts tumor suppression potentials by downregulating Snail in colorectal cancer DLD-1 and HCT116 cells, while it interacts with CK1ع to activate $\beta$-catenin/TCF signaling to promote cancer progress in colorectal cancer cells $(37,47)$. It is likely more reasonable to jointly consider multiple DDX3-associated proteins to analyze their influences on tumors and the patients OS.

Cancer subtypes. DDX3 has dissimilar expression and biological function in the specific histological subtypes of lung cancer $(34,35)$. DDX3 is overexpressed in some histopathologic subtypes of lung cancer, including small cell carcinoma, adenocarcinoma and squamous cell carcinoma, in which DDX3 functions as an oncogene (35). In contrast, DDX3 is considered to be a tumor suppressor in non-small cell lung cancer (34). However, the reason for this diversity needs further study. A different subtype of colorectal cancer cells has different sensitivity to the DDX3 inhibitor RK-33 (40). Thus, it is necessary to develop precision medicine and therapy for various subtypes of cancer patients based on the specific functions of DDX3.

Subcellular localization. The subcellular localization of DDX3 may shed light on controversial roles of DDX3 in different cancers. As a nuclear-cytoplasmic shuttling protein, DDX3 may have multifunctional roles in transcript regulation and protein synthesis to regulate various cellular processes. The nuclear localization of DDX3 is altered in cutaneous squamous cell carcinoma when compared with normal skin (15). In addition, DDX3 shows a less nuclear expression and more obvious cytoplasmic expression in OSCC tissues compared with the tissues adjacent to OSCC (41). Alteration of subcellular localization from nuclear to cytoplasm perhaps leads to complete loss of function of DDX3.

DDX3 mutation. DDX3 mutation is detected in several cancers, including medulloblastoma, natural killer/T-cell lymphoma and chronic lymphocytic leukemia (50-54). Obviously, the wild-type and mutant DDX3 may exhibit different functions in normal and cancer cells. The loss of DDX3 function by gene mutations results in tumor pathogenesis through altered RNA unwinding. Not only the expression level but also the gene mutation of DDX3 determines its functional divergence of DDX3.

\section{Potential anticancer drugs targeting DDX3}

DDX3 possesses several functional domains with kinase activities, which is designed as drug targets by docking small molecular inhibitors (72). In recent years, more and more novel small molecular inhibitors targeting DDX3 are developed as potential target chemical drugs for cancer therapy. We illustrated several chemical compounds by inhibiting DDX3 kinase activity, and their advantages and disadvantages are compared. Up to now, at least five types of compounds, including FE-15, NZ51, ketorolac salt, doxorubicin and RK-33, have been designed to inhibit DDX3 activity (Table II).

FE-15, the first small molecule by inhibiting ATPase activity of DDX3, was specifically designed to inhibit HIV-1 replication by targeting the RNA binding site of DDX3 (73). However, the main drawbacks of FE-15 are low activity and selectivity. It is difficult for FE-15 to selectively recognize DDX3 due to the high homology between other similar RNA helicases.

NZ51, one of ring-expanded nucleosides (REN), has been identified as a potential DDX3 inhibitor by abrogating the 


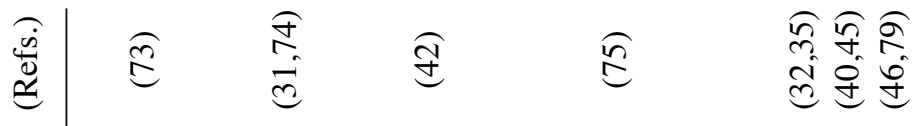

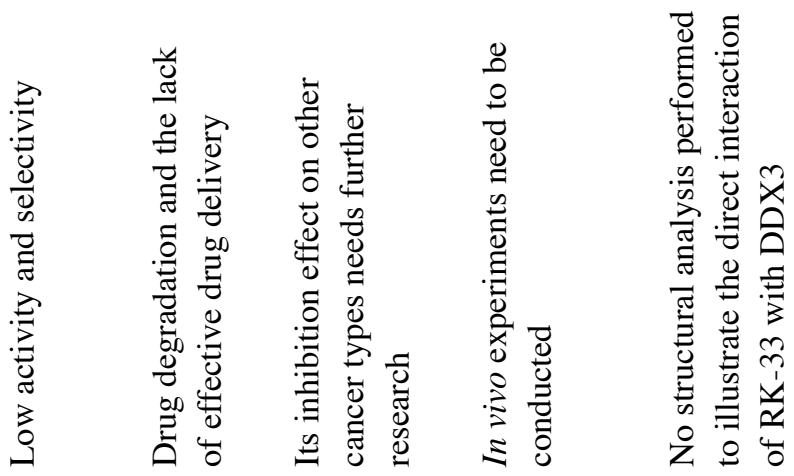

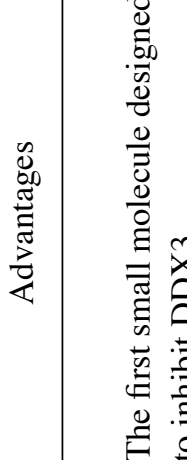

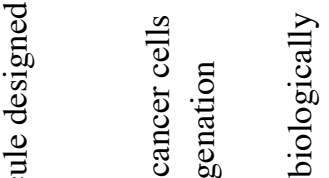

岂

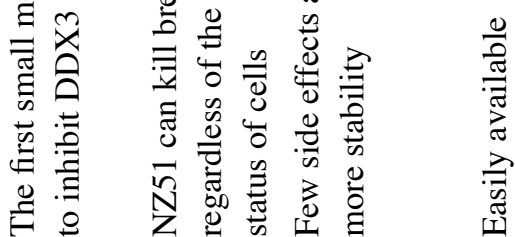

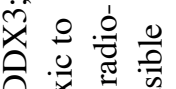

萡

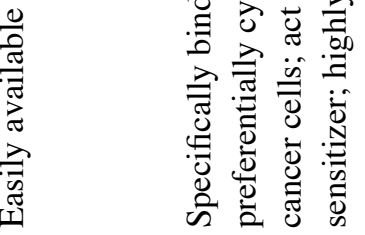

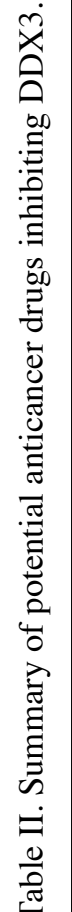

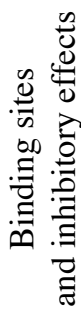

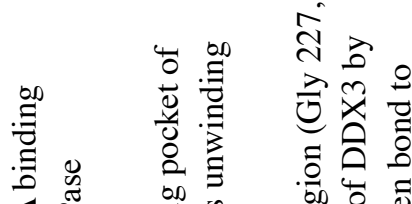

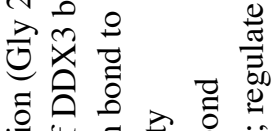

章 它

ప్ర.

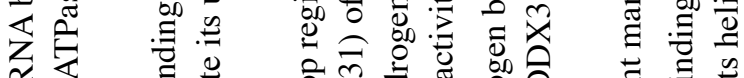
《

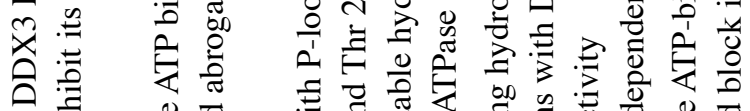

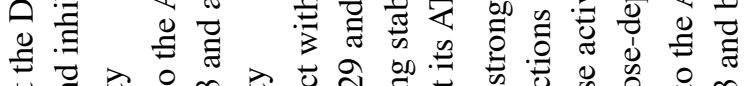

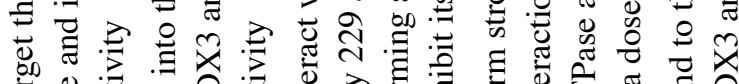

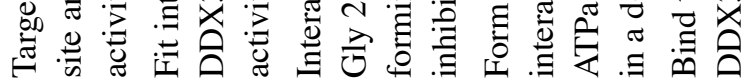

$\frac{\text { 해 }}{\overline{0}} \frac{\overline{0}}{0.00}$

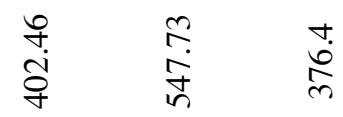

\begin{tabular}{ll}
$\infty$ & \multirow{J}{*}{} \\
\multirow{2}{*}{} & \multirow{7}{*}{} \\
in &
\end{tabular}

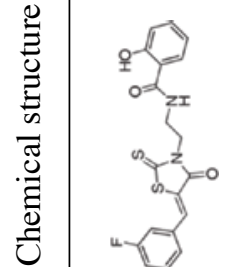

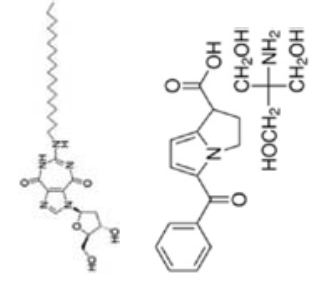

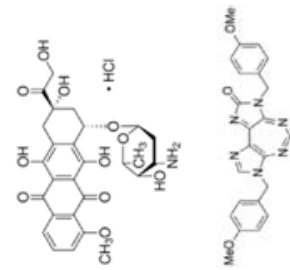

$\stackrel{\text { : }}{: \frac{0}{3}}$

$\frac{n}{\frac{1}{1}}$

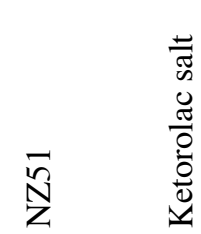

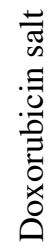

$\stackrel{m}{\mathscr{1}}$ 
unwinding activity of DDX3 helicase (74). NZ51 significantly inhibits cell motility and viability of breast cancer cells by targeting the ATP binding pocket domain of DDX3 (31). The compound NZ51 stays active in normoxic and hypoxic cell environments, so NZ51 can kill breast cancer cells regardless of cellular oxygenation status. Unfortunately, NZ51 has no significant tumor suppression efficiency on tumor-bearing mice (31). This is due to compound degradation of NZ51, which leads to lack of effective drug delivery in nude mice.

Ketorolac salt is a newly discovered bioactive compound against DDX3, which can be used as a candidate drug to treat oral cancer (42). Ketorolac salt interacts with the P-loop region (Gly 227, Gly 229 and Thr 231) of DDX3 by forming stable hydrogen bonds. It reduces cell viability by inhibiting the ATPase activity of DDX3 in a dose-dependent manner in OSCC cells. Compared with other chemical inhibitor molecules, ketorolac salt is a type of natural bioactive compound, which has few side effects with better biological stability. So far, the novel ketorolac salt-derived chemical compounds need to be further optimized to efficiently inhibit cancer cells.

A well-known antitumor drug, doxorubicin, has shown in vitro anticancer activity on OSCC cells by inhibiting cellular DDX3 ATPase activity (75). In addition, doxorubicin can form strong hydrogen bonds with DDX3. Although doxorubicin is easily available for treating cancer, the in vivo anticancer effects are accompanied by cardiotoxicity. A key issue is wheather DDX3 ATPase domain is the only drug target of doxorubicin in cancer cells.

Another small molecular inhibitor RK-33 has been demonstrated to bind with the ATP-binding domain of DDX3 to block its helicase activity in Ewing sarcoma, breast, colorectal, prostate and lung cancer cells $(32,35,40,45,46,79)$. RK-33 causes cell cycle G1 arrest and cell apoptosis in DDX3-overexpressing cancer cells. In addition, RK-33 suppresses DDX3 function by impairing Wnt signaling and inhibiting the non-homologous end joining (NHEJ) activity. RK-33 has a great many advantages as follows. Firstly, RK-33 specifically binds to DDX3, rather than the closely related DEAD-box proteins DDX5 and DDX17. Secondly, cell sensitivity to RK-33 is correlated with DDX3 protein expression levels. Therefore, RK-33 is preferentially cytotoxic to DDX3-overexpressing cancer cells. Thirdly, RK-33 has been reported to act as a radiosensitizer, which may reduce normal tissue toxicity by decreasing the radiation dose. However, no structural analysis exists to support the direct interaction of RK-33 with DDX3 to date. However, further studies are needed to optimize drug formulation, dose and delivery.

Among the inhibitors targeting DDX3, the most widely studied anticancer agent is RK-33, which has favorable preclinical anticancer activity. Women harboring breast cancer 1 (BRCA1) gene mutation are at high risk of suffering from breast cancer, due to a deficiency in homologous recombination to deal with DNA damage (76). This leads cancer cells to a greater dependency on the remaining repair pathways to deal with DNA damage. So BRCA1 mutation carriers are particularly sensitive to poly(ADP-ribose) polymerase (PARP) inhibitors, which inhibit the base excision repair (BER), a single-strand break repair pathway for DNA damage $(32,77)$. However, cell resistance against PARP inhibitors is widespread (32). RK-33 has been demonstrated to impair radiation-induced DNA damage repair by inhibiting NHEJ, a predominant mechanism for double-strand break repair $(35,78)$. Therefore, the combination treatment using RK-33 and PARP inhibitors has potential synergistic anticancer action and induces synthetic lethality in BRCA1 pro- and deficient cells (32). However, further human clinical trials are needed to evaluate the safety and efficacy of this combined treatment.

In contrast, DDX3 inhibitor RK-33 has been tried to be modify into a novel nanoparticle reagent for cancer. The poly (lactic-co-glycolic acid) (PLGA) encapsulated RK-33 nanoparticles are suitable for intravenous injection (79), which is a systemic delivery and controlled-release drug delivery (80). PLGA nanoparticles loading with RK-33 may facilitate to overcome chemical hydrophobicity of RK-33. RK-33-PLGA nanoparticles exhibit cytotoxicity to human breast carcinoma MCF-7 cells in vitro, and a systemic retention of RK-33 is markedly improved in mice (79).

Reports on chemical molecules designed to increase DDX3 expression to exert tumor suppression activity are still rare. Some p53 protein-stabilizing drugs including nutlin and bortezomib may benefit recovery of DDX3 expression to improve the clinical outcome of cancer patients (34).

\section{Future perspectives}

DDX3 has been confirmed to regulate the occurrence and development of cancers and viral infection. DDX3 is one of the inflammatory markers, which is correlated with tumorigenesis. Multiple factors interplay with DDX3 to confer its versatile functions.

Studies have shown that the intracellular mRNA and protein expression of DDX3 are inconsistent in some cancer cells. These findings suggest that post-translational modification may be quite important in DDX3 activity. Up to now, few studies have directly addressed the relationship between DDX3 modification and cancer progress. So it deserves great research attention in the future.

DDX3 shows its multiple enzyme activity including ATPase and RNA helicase. We should distinguish which activity or which functional domain is required for DDX3 functions to regulate cancer. Inducing mutation at the target enzyme site by CRISPR-Cas9 and constructing a truncated DDX3 protein may help us achieve this goal.

DDX3 is involved in multiple tumor-related signaling pathways. Recently, a combination treatment of DDX3 and PARP inhibitors induced cooperative effects in breast cancer. This is an indication that multi-target therapy based on DDX3 may achieve greater efficacy.

Several compounds have been developed to potentially decrease DDX3 activity to inhibit tumor growth. However, these compounds have their shortcomings. Nowadays, ligand-receptor interaction can be simulated with computer modeling and theoretical calculation methods. Moreover, 3D structures of DDX3 can provide useful information for learning its molecular function mechanism and designing drugs. The novel small molecule inhibitors targeting DDX3 can be developed and optimized according to the previous compounds. Nevertheless, the successful development of biomedical technologies could ultimately translate our understanding of DDX3 functions in cancer into strategies for the inhibition of cancer. 


\section{Acknowledgements}

The present study was financially supported by the grants from the National Key Basic Research Program of China (grant nos. 2013CB911303 and 2011CB910703), the National Natural Sciences Foundation of China (grant no. 31470810), the Science and Technology Department of Sichuan Province (grant no. 2017JY0232), the Health and Family Planning Commission of Sichuan Province (grant no. 17ZD045) and the Guangdong Innovative Research Team Program (grant no. 2011Y073).

\section{References}

1. Linder P and Jankowsky E: From unwinding to clamping - the DEAD box RNA helicase family. Nat Rev Mol Cell Biol 12: 505-516, 2011.

2. Linder $P$ and Fuller-Pace F: Happy birthday: 25 years of DEAD-box proteins. Methods Mol Biol 1259: 17-33, 2015.

3. Tarn WY and Chang TH: The current understanding of Ded1p/DDX3 homologs from yeast to human. RNA Biol 6: 17-20,2009.

4. Kim YS, Lee SG, Park SH and Song K: Gene structure of the human DDX3 and chromosome mapping of its related sequences. Mol Cells 12: 209-214, 2001.

5. Kotov AA, Olenkina OM, Godneeva BK, Adashev VE and Olenina LV: Progress in understanding the molecular functions of DDX3Y (DBY) in male germ cell development and maintenance. Biosci Trends 11: 46-53, 2017.

6. Rosner A and Rinkevich B: The DDX3 subfamily of the DEAD box helicases: Divergent roles as unveiled by studying different organisms and in vitro assays. Curr Med Chem 14: 2517-2525, 2007.

7. Rodamilans B and Montoya G: Expression, purification, crystallization and preliminary X-ray diffraction analysis of the DDX3 RNA helicase domain. Acta Crystallogr Sect F Struct Biol Cryst Commun 63: 283-286, 2007.

8. Högbom M, Collins R, van den Berg S, Jenvert RM, Karlberg T, Kotenyova T, Flores A, Karlsson Hedestam GB and Schiavone LH: Crystal structure of conserved domains 1 and 2 of the human DEAD box helicase DDX3X in complex with the mononucleotide AMP. J Mol Biol 372: 150-159, 2007.

9. Soto-Rifo R and Ohlmann T: The role of the DEAD-box RNA helicase DDX3 in mRNA metabolism. Wiley Interdiscip Rev RNA 4: 369-385, 2013.

10. Rocak S and Linder P: DEAD-box proteins: The driving forces behind RNA metabolism. Nat Rev Mol Cell Biol 5: 232-241, 2004.

11. Zhou Z, Licklider LJ, Gygi SP and Reed R: Comprehensive proteomic analysis of the human spliceosome. Nature 419: 182-185, 2002.

12. Fröhlich A, Rojas-Araya B, Pereira-Montecinos C, Dellarossa A, Toro-Ascuy D, Prades-Pérez Y, García-de-Gracia F, Garcés-Alday A, Rubilar PS, Valiente-Echeverría F, et al: DEAD-box RNA helicase DDX3 connects CRM1-dependent nuclear export and translation of the HIV-1 unspliced mRNA through its N-terminal domain. Biochim Biophys Acta 1859: 719-730, 2016

13. Yedavalli VS, Neuveut C, Chi YH, Kleiman L and Jeang KT: Requirement of DDX3 DEAD box RNA helicase for HIV-1 Rev-RRE export function. Cell 119: 381-392, 2004.

14. Lai MC, Lee YH and Tarn WY: The DEAD-box RNA helicase DDX3 associates with export mRNPs as well as TAP and participates in translational control. Mol Biol Cell 19: 3847-3858, 2008

15. Chao CH, Chen CM, Cheng PL, Shih JW, Tsou AP and Lee YH: DDX3, a DEAD box RNA helicase with tumor growthsuppressive property and transcriptional regulation activity of the p21waf1/cip1 promoter, is a candidate tumor suppressor. Cancer Res 66: 6579-6588, 2006.

16. Lee CS, Dias AP, Jedrychowski M, Patel AH, Hsu JL and Reed R: Human DDX3 functions in translation and interacts with the translation initiation factor eIF3. Nucleic Acids Res 36 4708-4718, 2008.

17. Shih JW, Tsai TY, Chao CH and Wu Lee YH: Candidate tumor suppressor DDX3 RNA helicase specifically represses cap-dependent translation by acting as an eIF4E inhibitory protein. Oncogene 27: 700-714, 2008.
18. Oh S, Flynn RA, Floor SN, Purzner J, Martin L, Do BT, Schubert S, Vaka D, Morrissy S, Li Y, et al: Medulloblastoma-associated DDX3 variant selectively alters the translational response to stress. Oncotarget 7: 28169-28182, 2016.

19. Sun M, Zhou T, Jonasch E and Jope RS: DDX3 regulates DNA damage-induced apoptosis and p53 stabilization. Biochim Biophys Acta 1833: 1489-1497, 2013.

20. Chang PC, Chi CW, Chau GY, Li FY, Tsai YH, Wu JC and Wu Lee YH: DDX3, a DEAD box RNA helicase, is deregulated in hepatitis virus-associated hepatocellular carcinoma and is involved in cell growth control. Oncogene 25: 1991-2003, 2006.

21. Chen CY, Chan CH, Chen CM, Tsai YS, Tsai TY, Wu Lee YH and You LR: Targeted inactivation of murine Ddx3x: Essential roles of Ddx3x in placentation and embryogenesis. Hum Mol Genet 25: 2905-2922, 2016.

22. Bol GM, Xie M and Raman V: DDX3, a potential target for cancer treatment. Mol Cancer 14: 188, 2015.

23. Wang $\mathrm{H}$ and Ryu WS: Hepatitis B virus polymerase blocks pattern recognition receptor signaling via interaction with DDX3: Implications for immune evasion. PLoS Pathog 6: e1000986, 2010.

24. Angus AG, Dalrymple D, Boulant S, McGivern DR, Clayton RF, Scott MJ, Adair R, Graham S, Owsianka AM, Targett-Adams P, et al: Requirement of cellular DDX3 for hepatitis $C$ virus replication is unrelated to its interaction with the viral core protein. J Gen Virol 9: 122-132, 2010.

25. Huang JS, Chao CC, Su TL, Yeh SH, Chen DS, Chen CT, Chen PJ and Jou YS: Diverse cellular transformation capability of overexpressed genes in human hepatocellular carcinoma. Biochem Biophys Res Commun 315: 950-958, 2004.

26. Li HK, Mai RT, Huang HD, Chou CH, Chang YA, Chang YW, You LR, Chen CM and Lee YH: DDX3 Represses stemness by epigenetically modulating tumor-suppressive miRNAs in hepatocellular carcinoma. Sci Rep 6: 28637, 2016.

27. Botlagunta M, Vesuna F, Mironchik Y, Raman A, Lisok A, Winnard P Jr, Mukadam S, Van Diest P, Chen JH, Farabaugh P, et al: Oncogenic role of DDX3 in breast cancer biogenesis. Oncogene 27: 3912-3922, 2008.

28. Botlagunta M, Krishnamachary B, Vesuna F, Winnard PT Jr, Bol GM, Patel AH and Raman V: Expression of DDX3 is directly modulated by hypoxia inducible factor-1 alpha in breast epithelial cells. PLoS One 6: e17563, 2011.

29. Bol GM, Raman V, van der Groep P, Vermeulen JF, Patel AH, van der Wall E and van Diest PJ: Expression of the RNA helicase DDX3 and the hypoxia response in breast cancer. PLoS One 8: e63548, 2013.

30. Heerma van Voss MR, Schrijver WA, Ter Hoeve ND, Hoefnagel LD, Manson QF, van der Wall E, Raman V and van Diest PJ; Dutch distant breast cancer metastases consortium: The prognostic effect of DDX3 upregulation in distant breast cancer metastases. Clin Exp Metastasis 34: 85-92, 2017.

31. Xie M, Vesuna F, Botlagunta M, Bol GM, Irving A, Bergman Y, Hosmane RS, Kato Y, Winnard PT Jr and Raman V: NZ51, a ring-expanded nucleoside analog, inhibits motility and viability of breast cancer cells by targeting the RNA helicase DDX3. Oncotarget 6: 29901-29913, 2015.

32. Heerma van Voss MR, Brilliant JD, Vesuna F, Bol GM, van der Wall E, van Diest PJ and Raman V: Combination treatment using DDX3 and PARP inhibitors induces synthetic lethality in BRCA1-proficient breast cancer. Med Oncol 34: 33, 2017.

33. Wu DW, Liu WS, Wang J, Chen CY, Cheng YW and Lee H: Reduced $\mathrm{p} 21^{\mathrm{WAF} 1 / \mathrm{CIP} 1}$ via alteration of $\mathrm{p} 53$-DDX3 pathway is associated with poor relapse-free survival in early-stage human papillomavirus-associated lung cancer. Clin Cancer Res 17: 1895-1905, 2011

34. Wu DW, Lee MC, Wang J, Chen CY, Cheng YW and Lee H: DDX3 loss by p53 inactivation promotes tumor malignancy via the MDM2/Slug/E-cadherin pathway and poor patient outcome in non-small-cell lung cancer. Oncogene 33: 1515-1526, 2014.

35. Bol GM, Vesuna F, Xie M, Zeng J, Aziz K, Gandhi N, Levine A, Irving A, Korz D, Tantravedi S, et al: Targeting DDX3 with a small molecule inhibitor for lung cancer therapy. EMBO Mol Med 7: 648-669, 2015

36. Su CY, Lin TC, Lin YF, Chen MH, Lee CH, Wang HY, Lee YC, Liu YP, Chen CL and Hsiao M: DDX3 as a strongest prognosis marker and its downregulation promotes metastasis in colorectal cancer. Oncotarget 6: 18602-18612, 2015.

37. He TY, Wu DW, Lin PL, Wang L, Huang CC, Chou MC and Lee H: DDX3 promotes tumor invasion in colorectal cancer via the CK1ع/Dvl2 axis. Sci Rep 6: 21483, 2016. 
38. Wu DW, Lin PL, Cheng YW, Huang CC, Wang L and Lee $\mathrm{H}$ : 'KRAS-induced tumor invasion in colorectal cancer via the $\beta$-catenin/ZEB1 axis. Oncotarget 7: 22687-22699, 2016.

39. Wu DW, Lin PL, Wang L, Huang CC and Lee H: The YAP1/SIX2 axis is required for DDX3-mediated tumor aggressiveness and cetuximab resistance in KRAS-wild-type colorectal cancer. Theranostics 7: 1114-1132, 2017.

40. Heerma van Voss MR, Vesuna F, Trumpi K, Brilliant J, Berlinicke C, de Leng W, Kranenburg O, Offerhaus GJ, Bürger $\mathrm{H}$, van der Wall $\mathrm{E}$, et al: Identification of the DEAD box RNA helicase DDX3 as a therapeutic target in colorectal cancer. Oncotarget 6: 28312-28326, 2015.

41. Lee CH, Lin SH, Yang SF, Yang SM, Chen MK, Lee H, Ko JL, Chen CJ and Yeh KT: Low/negative expression of DDX3 may predict poor prognosis in non-smoker patients with oral cancer. Oral Dis 20: 76-83, 2014.

42. Samal SK, Routray S, Veeramachaneni GK, Dash R and Botlagunta M: Ketorolac salt is a newly discovered DDX3 inhibitor to treat oral cancer. Sci Rep 5: 9982, 2015.

43. Heerma van Voss MR, van Kempen PM, Noorlag R, van Diest PJ, Willems SM and Raman V: DDX3 has divergent roles in head and neck squamous cell carcinomas in smoking versus non-smoking patients. Oral Dis 21: 270-271, 2015.

44. Stransky N, Egloff AM, Tward AD, Kostic AD, Cibulskis K, Sivachenko A, Kryukov GV, Lawrence MS, Sougnez C, McKenna A, et al: The mutational landscape of head and neck squamous cell carcinoma. Science 333: 1157-1160, 2011.

45. Wilky BA, Kim C, McCarty G, Montgomery EA, Kammers K, DeVine LR, Cole RN, Raman V and Loeb DM: RNA helicase DDX3: A novel therapeutic target in Ewing sarcoma. Oncogene 35: 2574-2583, 2016.

46. Xie M, Vesuna F, Tantravedi S, Bol GM, Heerma van Voss MR, Nugent K, Malek R, Gabrielson K, van Diest PJ, Tran PT and Raman V: RK-33 Radio sensitizes prostate cancer cells by blocking the RNA helicase DDX3. Cancer Res 76: 6340-6350, 2016.

47. Sun M, Song L, Zhou T, Gillespie GY and Jope RS: The role of DDX3 in regulating Snail. Biochim Biophys Acta 1813: 438-447, 2011.

48. Liang S, Yang Z, Li D, Miao X, Yang L, Zou Q and Yuan Y: The clinical and pathological significance of nectin-2 and DDX3 expression in pancreatic ductal adenocarcinomas. Dis Markers 2015: 379568, 2015.

49. Miao X, Yang ZL, Xiong L, Zou Q, Yuan Y, Li J, Liang L, Chen $M$ and Chen S: Nectin-2 and DDX3 are biomarkers for metastasis and poor prognosis of squamous cell/adenosquamous carcinomas and adenocarcinoma of gallbladder. Int J Clin Exp Pathol 6: 179-190, 2013.

50. Pugh TJ, Weeraratne SD, Archer TC, Pomeranz Krummel DA, Auclair D, Bochicchio J, Carneiro MO, Carter SL, Cibulskis K, Erlich RL, et al: Medulloblastoma exome sequencing uncovers subtype-specific somatic mutations. Nature 488: 106-110, 2012.

51. Robinson G, Parker M, Kranenburg TA, Lu C, Chen X, Ding L, Phoenix TN, Hedlund E, Wei L, Zhu X, et al: Novel mutations target distinct subgroups of medulloblastoma. Nature 488: 43-48, 2012.

52. Jiang L, Gu ZH, Yan ZX, Zhao X, Xie YY, Zhang ZG, Pan CM, $\mathrm{Hu}$ Y, Cai CP, Dong Y, et al: Exome sequencing identifies somatic mutations of DDX3X in natural killer/T-cell lymphoma. Nat Genet 47: 1061-1066, 2015.

53. Wang L, Lawrence MS, Wan Y, Stojanov P, Sougnez C, Stevenson K, Werner L, Sivachenko A, DeLuca DS, Zhang L, et al: SF3B1 and other novel cancer genes in chronic lymphocytic leukemia. N Engl J Med 365: 2497-2506, 2011.

54. Ojha J, Secreto CR, Rabe KG, Van Dyke DL, Kortum KM, Slager SL, Shanafelt TD, Fonseca R, Kay NE and Braggio E: Identification of recurrent truncated DDX3X mutations in chronic lymphocytic leukaemia. Br J Haematol 169: 445-448, 2015.

55. Cruciat CM, Dolde C, de Groot RE, Ohkawara B, Reinhard C, Korswagen HC and Niehrs C: RNA helicase DDX3 is a regulatory subunit of casein kinase 1 in Wnt- $\beta$-catenin signaling. Science 339: 1436-1441, 2013.

56. Chen HH, Yu HI, Cho WC and Tarn WY: DDX3 modulates cell adhesion and motility and cancer cell metastasis via Rac1mediated signaling pathway. Oncogene 34: 2790-2800, 2015

57. Chen B, Zeng X, He Y, Wang X, Liang Z, Liu J, Zhang P, Zhu H, $\mathrm{Xu} N$ and Liang S: STC2 promotes the epithelial-mesenchymal transition of colorectal cancer cells through AKT-ERK signaling pathways. Oncotarget 7: 71400-71416, 2016.

58. Wang Y, Shi J, Chai K, Ying X and Zhou BP: The role of snail in EMT and tumorigenesis. Curr Cancer Drug Targets 13: 963-972, 2013.
59. Zhou BP, Deng J, Xia W, Xu J, Li YM, Gunduz M and Hung MC: Dual regulation of Snail by GSK-3beta-mediated phosphorylation in control of epithelial-mesenchymal transition. Nat Cell Biol 6: 931-940, 2004.

60. Sun M, Song L, Li Y, Zhou T and Jope RS: Identification of an antiapoptotic protein complex at death receptors. Cell Death Differ 15: 1887-1900, 2008.

61. Wang SP, Wang WL, Chang YL, Wu CT, Chao YC, Kao SH, Yuan A, Lin CW, Yang SC, Chan WK, et al: p53 controls cancer cell invasion by inducing the MDM2-mediated degradation of Slug. Nat Cell Biol 11: 694-704, 2009.

62. Chen B, Li H, Zeng X, Yang P, Liu X, Zhao X and Liang S: Roles of microRNA on cancer cell metabolism. J Transl Med 10: 228, 2012.

63. Chen Y, Fu LL, Wen X, Liu B, Huang J, Wang JH and Wei YQ: Oncogenic and tumor suppressive roles of microRNAs in apoptosis and autophagy. Apoptosis 19: 1177-1189, 2014.

64. Ha M and Kim VN: Regulation of microRNA biogenesis. Nat Rev Mol Cell Biol 15: 509-524, 2014.

65. Zhao L, Mao Y, Zhao Y and He Y: DDX3X promotes the biogenesis of a subset of miRNAs and the potential roles they played in cancer development. Sci Rep 6: 32739, 2016.

66. Valiente-Echeverría F, Hermoso MA and Soto-Rifo R: RNA helicase DDX3: At the crossroad of viral replication and antiviral immunity. Rev Med Virol 25: 286-299, 2015.

67. Soulat D, Bürckstümmer T, Westermayer S, Goncalves A, Bauch A, Stefanovic A, Hantschel O, Bennett KL, Decker T and Superti-Furga G: The DEAD-box helicase DDX3X is a critical component of the TANK-binding kinase 1-dependent innate immune response. EMBO J 27: 2135-2146, 2008.

68. Gu L, Fullam A, Brennan R and Schröder M: Human DEAD box helicase 3 couples IкB kinase $\varepsilon$ to interferon regulatory factor 3 activation. Mol Cell Biol 33: 2004-2015, 2013.

69. Wang X, Wang R, Luo M, Li C, Wang HX, Huan CC, Qu YR, Liao Y and Mao X: (DEAD)-box RNA helicase 3 modulates $\mathrm{NF}-\kappa \mathrm{B}$ signal pathway by controlling the phosphorylation of PP2A-C subunit. Oncotarget 8: 33197-33213, 2017.

70. Xiang N, He M, Ishaq M, Gao Y, Song F, Guo L, Ma L, Sun G, Liu D, Guo D and Chen Y: The DEAD-box RNA helicase DDX3 interacts with NF- $\kappa$ B subunit p65 and suppresses p65-mediated transcription. PLoS One 11: e0164471, 2016.

71. Dunford A, Weinstock DM, Savova V, Schumacher SE, Cleary JP, Yoda A, Sullivan TJ,Hess JM, Gimelbrant AA, Beroukhim R, et al: Tumor-suppressor genes that escape from $\mathrm{X}$-inactivation contribute to cancer sex bias. Nat Genet 49: 10-16, 2017.

72. Backus KM, Correia BE, Lum KM, Forli S, Horning BD, González-Páez GE, Chatterjee S, Lanning BR, Teijaro JR, Olson AJ, et al: Proteome-wide covalent ligand discovery in native biological systems. Nature 534: 570-574, 2016.

73. Radi M, Falchi F, Garbelli A, Samuele A, Bernardo V, Paolucci S, Baldanti F, Schenone S, Manetti F, Maga G and Botta M: Discovery of the first small molecule inhibitor of human DDX3 specifically designed to target the RNA binding site: Towards the next generation HIV-1 inhibitors. Bioorg Med Chem Lett 22: 2094-2098, 2012.

74. Yedavalli VS, Zhang N, Cai H, Zhang P, Starost MF, Hosmane RS and Jeang KT: Ring expanded nucleoside analogues inhibit RNA helicase and intracellular human immunodeficiency virus type 1 replication. J Med Chem 51: 5043-5051, 2008.

75. Botlagunta M, Kollapalli B, Kakarla L, Gajarla SP, Gade SP, Dadi CL, Penumadu A and Javeed S: In vitro anti-cancer activity of doxorubicin against human RNA helicase, DDX3 Bioinformation 12: 347-353, 2016.

76. Bianchini G, Balko JM, Mayer IA, Sanders ME and Gianni L: Triple-negative breast cancer: Challenges and opportunities of a heterogeneous disease. Nat Rev Clin Oncol 13: 674-690, 2016.

77. TuttA,RobsonM,GarberJE,DomchekSM,AudehMW,WeitzelJN, Friedlander M, Arun B, Loman N, Schmutzler RK, et al: Oral poly(ADP-ribose) polymerase inhibitor olaparib in patients with BRCA1 or BRCA2 mutations and advanced breast cancer: A proof-of-concept trial. Lancet 376: 235-244, 2010.

78. Schwertman P, Bekker-Jensen S and Mailand N: Regulation of DNA double-strand break repair by ubiquitin and ubiquitin-like modifiers. Nat Rev Mol Cell Biol 17: 379-394, 2016.

79. Bol GM, Khan R, Heerma van Voss MR, Tantravedi S, Korz D, Kato Y and Raman V: PLGA nanoparticle formulation of RK-33: An RNA helicase inhibitor against DDX3. Cancer Chemother Pharmacol 76: 821-827, 2015 .

80. Danhier F, Lecouturier N, Vroman B, Jérôme C, MarchandBrynaert J, Feron O and Préat V: Paclitaxel-loaded PEGylated PLGA-based nanoparticles: In vitro and in vivo evaluation. J Control Release 133: 11-17, 2009. 